\title{
A Second Look at Chelation Therapy During COVID-19 as A Viable Option for Treating Cardiac Endothelial Dysfunction
}

\author{
Kessack $\mathbf{M}^{*}$ \\ College of Art and Sciences, University of Arizona Global Campus, USA
}

*Corresponding author: Kessack M, College of Art and Sciences, University of Arizona Global Campus, USA.

\section{Opinion}

Across the United States and the world, COVID-19 has altered the manner patients are treated and admitted to the hospital. Through this pandemic, bed space has been allocated for the massive on-rush of patients requiring acute assistance with ventilations and survival. There has been a $60 \%$ reduction in nonemergent surgeries being postponed due to lack of available beds, due to precautions for COVID exposure, and due to decreased staff available for non-COVID patients [1-3,]. Added to this mix is the fact that patients with pre-existing coronary artery disease (CAD) are more at risk for cardiac injury and complications if exposed to the coronavirus [4]. According to [4] complications from COVID include myocarditis, arrhythmia, and heart failure and are observed in CAD patients (as a result of the COVID virus) and nonCAD patients alike and are permanent. This is due, in part, to the cytokine release syndrome seen in many COVID patients (Ganatra, et al., 2020). The population without CAD issues can simply avoid exposure to the virus to ensure that they will not develop the CAD symptoms; however, the patients with pre-existing CAD may have their conditions exacerbated. Traditional therapies for myocarditis, arrhythmia, and heart failure could entail hospitalization with IV antibiotics, cardiac procedures entailing angiograms, with the result of in-hospital admissions for monitoring and treatment [5-7]. Medical intervention and treatment measures after exacerbation have demonstrated they are significantly more expensive than prevention measures [8].

Many studies have been conducted and published regarding the benefits of chelation therapy. One of the first studies demonstrated the effects of chelation therapy on improving cardiac patient symptoms and electrocardiographic improvement [9]. Later studies have further shown that contributing to cardiovascular disease are xenobiotic metals (i.e., lead, cadmium, mercury, arsenic) which in elevated levels demonstrates that a there is a correlation between the presence of heavy metals that help to initiate CAD symptoms and contributed to increased mortality rates from heart disease [10-13]. Studies conducted on the general population have demonstrated that these metals are in our environment and the population is constantly exposed to the heavy metals. Houston (2007) linked xenobiotic metals with myocardial infarction. Peguero and associates (2015) in a TACT study demonstrated that chelation therapy significantly reduced the presence of xenobiotic metals in participants with CAD who were treated for a myocardial infarction at least 6 weeks prior to the chelation therapy. The study encompassed 1708 patients who were randomized into a placebo group and a chelation group. Participants in this study were both male and female patients with a median age of 65 .

In light of the COVID pandemic we are presently experiencing, and with the studies demonstrating reduced symptoms and EKG arrhythmia improvement, chelation therapy might bring a needed alternative method to CAD patients to prevent exacerbations in their condition and decrease hospital admissions. Additional benefits of chelation therapy have also been demonstrated on diabetic patients who are also at risk for developing cardiac illness and peripheral artery disease (PAD) [14-16]. Chelation therapy through current quantitative experimental research studies are demonstrating 
that this therapy should be reviewed and considered for a viable option for CAD patients to prevent disease exacerbations and hospitalizations currently and in the future.

\section{Acknowledgement}

None.

\section{Conflict of Interest}

No conflict of interest.

\section{References}

1. Ahmed Al-Jabir, Ahmed Kerwan, Maria Nicola, Zaid Alsafi, Mehdi Khan, et al. (2020) Impact of the Coronavirus (COVID-19) pandemic on surgical practice - Part 2 (surgical prioritisation). Int J Surg 79: 233248.

2. Centers for Disease Control and Prevention (2020) Healthcare facility guidance.

3. Ahmet Surek, Sina Ferahman, Eyup Gemici, Ahmet Cem Dural, Turgut Donmez, et al. (2020) Effects of COVID-19 pandemic on general surgical emergencies: Are some emergencies really urgent? Level 1 trauma center experience. Eur J Trauma Emerg Surg pp. 1-6.

4. Sarju Ganatra, Sourbha S Dani, Sachin Shah, Aarti Asnani, Tomas G Neilan, et al. (2020) Management of Cardiovascular Disease During Coronavirus Disease (COVID-19) Pandemic. Trends Cardiovasc Med 30(6): 315-325.

5. Steven M Hollenberg, Lynne Warner Stevenson, Tariq Ahmad, Vaibhav J Amin, Biykem Bozkurt, et al. (2019) 2019 ACC expert consensus decision pathway on risk assessment, management, and clinical trajectory of patients hospitalized with heart failure: A report of the American College of Cardiology solution set oversight committee. J Am Coll Cardiol 74(15): 1966-2011.

6. Jason C Schultz, Anthony A Hilliard, Leslie T Cooper Jr, Charanjit S Rihal (2009) Diagnosis and treatment of viral myocarditis. Mayo Clin Proc 84(11): 1001-1009.
7. Tschöpe C, Cooper LT, Torre-Amione G, Van Linthout S (2019) Management of myocarditis-related cardiomyopathy in adults. Circ Res 124: 1568-1583.

8. Musich S, Wang S, Hawkins K, Klemes A (2016) The impact of personalized preventive care on health care quality, utilization, and expenditures. Popul Health Manag 19(6): 389-397.

9. Clarke CN, Clarke NE (1956) Treatment of angina pectoris with disodium ethylene diamine tetraacetic acid. Am J Med Sci 6(232): 654666.

10. Lustberg M, Silbergeld E (2002) Blood levels and mortality. Archives of Internal Medicine 162: 2443-2449.

11. Nawrot T, Thijs L, Den Hond E, Roles H, Staessen J (2002) An epidemiological reappraisal of the association between blood pressure and blood lead: A metaanalysis. J Hum Hypertens 16: 123-131.

12. JL Pirkle, J Schwartz, JR Landis, WR Harlan (1985) The relationship between blood lead levels and blood pressure and its cardiovascular risk implications. Am J Epidemiol 121: 246-258.

13. Tellez-Plaza M, Navas-Acien A, Crainiceanu CM, Guallar E (2008) Cadmium exposure and hypertension in the 1999-2004. National Health and Nutrition Examination Survey (NHANES). Environ Health Perspect 116: 51-56.

14. Diaz D, Fonseca V, Aude YW, Lamas GA (2018) Chelation therapy to prevent diabetes-associated cardiovascular events.

1. Curr Opin Endocrinol Diabetes Obes 25(4): 258-266.

15. Lamas G (2019) Chelation therapy in diabetic patients with critical limb ischemia (TACT-PAD). Mount Sinai Medical Center, Miami FL. CinicalTrials.gov Identifier NCT03424746.

16. Houston MC (2007) The role of mercury and cadmium heavy metals in vascular disease, hypertension, coronary heart disease, and myocardial infarction. Altern Ther Health Med 13(2): 128-133. 